\title{
Frischer Wind aus Europa
}

VON TINA HOFMANN UND ANUSCHKA NOVAKOVIC

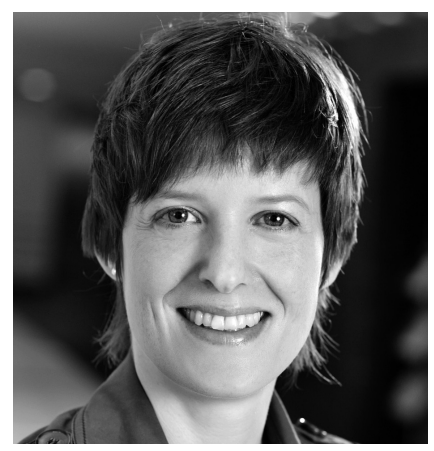

Tina Hofmann ist Diplom-Verwaltungswissenschaftlerin und seit 2004 beim Paritätischen Gesamtverband. Sie leitet das Referat für Arbeitsmarkt- und Sozialpolitik. arbeitsmarkt@paritaet.org

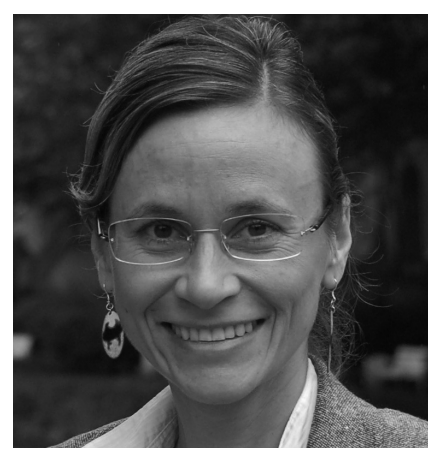

Anuschka Novakovic, LL.M. ist Rechtsanwältin. Seit 2011 arbeitet sie beim Paritätischen Gesamtverband und betreut dort das Referat "Grundlagen der Finanzierung", das den Fachbereich Vergaberecht einschließt.

pflegesatz@paritaet.org

\author{
Insbesondere bei Dienstleitungen rund um \\ den Arbeitsmarkt hat die Vergabepraxis in den \\ letzten Jahren für Verschlechterungen für Nutzer, \\ Leistungsanbieter und Beschäftigte gesorgt. \\ Neue Initiativen aus der Europäischen Union \\ könnten nun bald Verbesserungen bringen.
}

In den letzten Jahren hat jede Bundesregierung ihre eigene Reform in der Arbeitsmarktförderung durchgesetzt. Meist mit einem hohen Qualitätsanspruch versehen, wurden Förderinstrumente gestrichen, zusammengelegt oder neu eingeführt.

Die jüngste Gesetzesreform verfolgte mit der Zulassung von Trägern und Maßnahmen das Ansinnen, eine durchgängig gute Qualität in der Arbeitsmarktförderung zu gewährleisten. Die Kritik der Wohlfahrtsverbände, dass eine wirkliche Qualitätsverbesserung in der Arbeitsförderung solange zum Scheitern verurteilt ist, wie man an der Praxis öffentlicher Ausschreibungen festhält, ist im Gesetzgebungsverfahren unerhört geblieben.

Zunehmend werden sogar die Arbeitsfördermaßnahmen für Menschen mit Behinderungen öffentlich ausgeschrieben. Das hat negative Konsequenzen für die Arbeitsförderung. Unter den Bedingungen des Monopoleinkaufs der Bundesagentur für Arbeit herrscht ein so massiver Preisdruck vor, dass die Mitarbeiterinnen und Mitarbeitern meist nicht leistungsgerecht vergütet werden können und tarifliche Standards unterlaufen werden. Daran ändert auch der seit 1. August 2012 gültige Mindestlohn in der Aus- und Weiterbildungsbranche nichts.

Die Wohlfahrtsverbände hoffen für ihre Anliegen zur Vergabepraxis auf neue Impulse der Europäischen Union. Sie hat zwei Richtlinienentwürfe zur Modernisierung des öffentlichen
Auftragswesens auf den Weg gebracht. Sobald die Richtlinienentwürfe im Europäischen Parlament verabschiedet sind, muss die Bundesregierung für eine Umsetzung der Vorgaben im deutschen Vergaberecht sorgen. Fest steht, dass in der deutschen Sozialwirtschaft nur der Richtlinienentwurf zur öffentlichen Auftragsvergabe relevant wird, und zwar nur für solche sozialen Dienstleistungen, die im Wege öffentlicher Aufträge vergeben werden. Das sind die sogenannten Arbeitsmarktdienstleistungen. Alle anderen sozialen Dienstleistungen werden typischerweise im sozialrechtlichen Dreiecksverhältnis erbracht und unterliegen damit nicht dem Vergaberecht.

Der Richtlinienentwurf zur öffentlichen Auftragsvergabe will die Strategie »Europa 2020« zur Maßgabe für die Auftragsbeschaffung staatlicher Stellen machen. Nach diesem Plan sollen die europäischen Volkswirtschaften wachsen und ihre Wettbewerbsfähigkeit auf den Weltmärkten behaupten, aber auch ressourcen- und umweltschonend vorgehen und den sozialen Zusammenhalt wahren. Die öffentliche Auftragsvergabe soll dabei als Instrument bereitstehen, gemeinsame gesellschaftliche Ziele zu unterstützen, wie etwa »bestmögliche Bedingungen für die Erbringung hochwertiger sozialer Dienstleistungen « schaffen. Einem Paukenschlag kommt die Aussage der Europäischen Union gleich, die üblichen Vergabeverfahren für soziale Dienstleistungen und ihre Besonderheiten seien ungeeignet. 
Deshalb können nun bei allen Auftragsvergaben staatliche Stellen stärker als bisher die Einhaltung von sozialen Kriterien bei der Auftragsausführung berücksichtigen. Beispielsweise kann die Beschäftigung von Langzeitarbeitslosen zur Voraussetzung der Auftragsvergabe gemacht werden. Erhalten bleibt im Kern die spezielle Auftragsvergabe an Werkstätten für Menschen mit Behinderungen. Überdies gilt künftig für alle Dienstleistungsaufträge: Die Auftraggeber dürfen bei der Angebotswertung die (bessere) Organisation, Qualifizierung und Erfahrung des für die Auftragsausführung eingesetzten Personals berücksichtigen. Es ist weiterhin möglich, aber nicht zwingend, die Zahlung gesetzlicher Mindestlöhne vorauszusetzen. Die Vorgabe einer Tarifbindung hingegen dürfte weiter unzulässig bleiben.

Der Richtlinienentwurf enthält darüber hinaus ein eigenes Kapitel über "Soziale und andere besondere Dienstleistungen «. Die Europäische Union erkennt den kulturellen Kontext der sozialen Dienstleistungen und ihren sensiblen Charakter an und schafft deshalb den Rahmen für spezielle Vergaberegelungen. Die Union verlangt, dass in den Vergabeverfahren den Besonderheiten der sozialen Dienstleistungen ausreichend Rechnung getragen werden kann. $\mathrm{Zu}$ den Besonderheiten zählen laut des Richtlinienentwurfs die Notwendigkeit, die Qualität, Kontinuität, Zugänglichkeit, Verfügbarkeit und Vollständigkeit der Dienstleistungen sicherzustellen sowie die spezifischen Bedürfnisse verschiedener Nutzerkategorien, die Einbeziehung und Ermächtigung der Nutzer sowie der Aspekt der Innovation. Bei einem Auftragswert (Schwellenwert) von unter 500.000 Euro soll es zwar keinerlei Verfahrensvorschriften geben. Stets müssen sich die Mitgliedsstaaten aber an einige Grundregeln halten und für Transparenz im Vergabeverfahren und Gleichbehandlung sorgen.

Sobald die Richtlinien im Laufe des Jahres vom Europäischen Parlament verabschiedet worden sind, steht eine Diskussion über die konkrete Umsetzung in deutsches Recht bevor. Dem deutschen Gesetzgeber wird ein breiter Ermessensspielraum zustehen, wie er die Regelungen ausgestaltet.

Vor diesem Hintergrund werden sich die Wohlfahrtsverbände für das Gesetzgebungsverfahren positionieren, wobei einige Aspekte eine Rolle spielen dürften: Dreh- und Angelpunkt für die Sicherung einer guten Qualität der Arbeitsmarktdienstleistungen ist eine Vergabe zu höheren Preisen, die eine deutlich bessere und damit angemessene Bezahlung der Fachkräfte in der Arbeitsförderung ermöglicht. Qualifiziertes und motiviertes Personal, das nicht nur kurzeitig in der Arbeitsförderung beschäftigt ist, kann nur so gewonnen und gehalten werden.

Soweit die Europäische Union die Zugänglichkeit und Verfügbarkeit sozialer Dienstleistungen sichergestellt sehen möchte, rückt sie vom Wettbewerbsgedanken als Primat der Leistungserbringung ab und nennt Prinzipien, die auch für das deutsche Sozialrecht prägend sind. Allerdings gibt es im Vorfeld des Einkaufs von Arbeitsmarktdienstleistungen keine Bedarfsplanung und Analyse, wie sie etwa im Rahmen der kommunalen Sozialplanung erfolgt. Es ist zu überlegen, die Vergabe von Arbeitsmarktdienstleistungen durch einige Elemente der Sozialplanung zu verbessern. Das wäre vor allem die stärkere Ausrichtung des Einkaufs anhand regional und nicht etwa zentral festgestellter Bedarfe, die Berücksichtigung der Bedarfe aus Sicht der Nutzerinnen und Nutzer und die ressort- und fachübergreifende Abstimmung der Maßnahmen der Arbeitsförderung, etwa an der Schnittstelle zur Jugend- oder Suchthilfe.

Damit Förderleistungen rechtzeitig angeboten werden können, ist die Vergabe zu flexibilisieren. Die Wohlfahrtsverbände haben mehrfach kritisiert, dass öffentliche Ausschreibungen so lange Vorlaufzeiten haben, dass ein kurzfristig entstehender Bedarf kaum gedeckt werden kann. Die Terminierung der Bundesagentur von Fördermaßnahmen an Schulen liegt häufig quer zu schulischen Planungszeiträumen, so dass die notwendige Abstimmung nicht möglich ist.

Diese Defizite legen es nahe, zur rechtzeitigen und ausreichenden Bereitstellung sozialer Dienstleistungen verstärkt auf beschränkte Ausschreibungen oder die freihändige Vergabe zurückzugreifen. Völlig neue Wege sind auch nötig, um dem von der Europäischen Union anerkannten Bedürfnis zu entsprechen, die Nutzerinnen und Nutzer sozialer Dienstleistungen »einzubeziehen und zu ermächtigen«. Zur ihrer »Einbeziehung« wäre es erforderlich, ihnen echte
Wunsch- und Wahlrechte einzuräumen, damit sie an der Auswahl passender soziale Dienstleistungen beteiligt werden. Ihre »Ermächtigung « würde wohl noch weitaus mehr erfordern. Hierfür müssten sie ein Stück weit die Definitionsmacht darüber erhalten, wie die Arbeitsfördermaßnahme abläuft. Das könnte beispielsweise bedeuten, auf ein ansonsten obligatorisches Bewerbungstraining im Einzelfall zu verzichten, weil das von dem Erwerbslosen nicht angenommen wird.

Damit Innovationen möglich sind, wäre anstelle öffentlicher Ausschreibungen über die Anwendung dialogorientierter Vergabeverfahren nachzudenken, wie sie in anderen Bereichen durch öffentliche Auftraggeber, beispielsweise bei Bauleistungen, erfolgreich praktiziert werden. Bei öffentlichen Ausschreibungen entwickeln und definieren die Auftraggeber alleine und eigenständig die einzukaufenden Leistungen. Soweit freie Träger innovative Projektideen an die Bundesagentur für Arbeit herantragen, müssen sie damit rechnen, bei deren anschließenden öffentlichen Ausschreibung ihres Konzepts keinen Zuschlag zu erhalten. Demgegenüber ermöglicht ein Dialogverfahren einen gemeinsamen Ideen- und Entwicklungsprozess.

\section{Resümee: Bewegung erwünscht}

Insgesamt wird deutlich, dass eine den sozialen Dienstleistungen angepasste Vergabe grundlegend neue Regelungen im Vergaberecht und eine neue Vergabepraxis der Bundesagentur für Arbeit und der Jobcenter erfordert. $\mathrm{Ob}$ sich dazu der notwendige politische Wille der Bundesregierung gewinnen lässt, ist derzeit offen. Immerhin haben selbst die Koalitionsfraktionen am 26. Juni 2012 einen Antrag in den Deutschen Bundestag eingebracht und darin gefordert, die Qualität vor allem auch bei Vergabe von Arbeitsmarktdienstleistungen stärker zu berücksichtigen. Seine Beratung im Bundestag steht nach der Sommerpause an. Es ist zu hoffen, dass die Parlamentarier von dem frischen Wind aus Europa erfasst und sich für eine neue Vergabepolitik in Deutschland entscheiden werden. 